\title{
A Novel FCM Algorithm Incorporating Spatial Information for Color Image Segmentation
}

\author{
Li Ling, Song Yingwei, Yin Zhongnan and Yang Xiuhua \\ College of Communication Engineering, Jilin University, Changchun 130012, \\ China \\ liling2002@jlu.edu.cn
}

\begin{abstract}
Fuzzy c-means clustering (FCM) with spatial information (FCM_S) is an effective algorithm for image segmentation. However, the FCM_S algorithm is not used for color image segmentation and also it produces over-segmentation results. In this paper, we present a novel fuzzy c-means algorithm named nFCM_S that incorporates spatial information into the membership function and cluster center function for segmentation of color images. Firstly, HSV color space is used for decomposition of color images. Then, to label the data points reliably, a linearly-weighted sum image is calculated on each $H S V$ component before clustering process. Finally, spatial information is incorporated in the standard FCM algorithm and $n F C M \_S$ is applied separately on each component of $H S V$ color space. Experiment results have shown that the $n F C M \_S$ algorithm achieves competitive segmentation results compared to other FCM-based algorithms.
\end{abstract}

Keywords: Fuzzy c-means, over-segmentation, spatial information, image segmentation, HSV color space

\section{Introduction}

Image segmentation plays an important role in variety of applications such as robot vision, object recognition, geographical imaging and medical imaging [1-6]. In general, fuzzy segmentation methods, especially the FCM algorithm, have been widely used in image segmentation [7-9]. Because of the introduction of fuzzy membership for each pixel, FCM can retain more information from the original image compared to the crisp or hard segmentation methods [10]. However, as a clustering method, FCM ignores spatial information and works only on grayscale images. Therefore, the standard FCM algorithm often produces over-segmentation so that disconnected areas of the image are clustered together and incomplete regions are produced.

Recently, many approaches incorporating spatial information have been used to overcome the over-segmentation problem. Weiling Cai [11] presented a FGFCM framework for medical image segmentation, which incorporated local spatial and grayscale information together. However, FGFCM framework produces over-segmentation. Lianghua he [12] proposed an improved FCM with spatial constraints (iFCM_S) to allow the labeling of a pixel to be influenced by the labels of its neighboring pixels. However, the problem of iFCM_S is that it can't be straightforwardly extended to segment color images.

Although many improved segmentation algorithms have been proposed, FCM has some limitations including ignoring spatial information, working only on grayscale images, and producing over-segmentation for color images. Thus, we propose the nFCM_S algorithm to segment color images efficiently. The differences of our method from that of others are as follows: Firstly, R, G, and B information is extracted from color images, and RGB color space is transformed to HSV model. Secondly, the function of input data is modified to relabel the pixels, and the modified function is applied on each 
HSV component. Thirdly, we modify fuzzy membership function and clustering center function to incorporate spatial information into FCM so that the nFCM_S algorithm can ameliorate the segmentation results.

The rest of this paper is organized as follows: the standard FCM is briefly introduced in Section 2. The FCM_S algorithm is described in detail in Section 3. In Section 4, a novel FCM-based clustering algorithm (nFCM_S) incorporating spatial information for color image segmentation is proposed. Then, in Section 5, experimental results on color images are presented. Finally, conclusions are made in Section 6.

\section{Fuzzy C-Means}

The standard FCM is proposed by Bezdek [13], which attracts more and more attention due to the introduction of the fuzzy set theory. The standard FCM generates fuzzy memberships of the data and assigns pixels to each cluster by fuzzy memberships. The standard FCM is an iterative optimization that minimizes the following objective function:

$$
J=\sum_{i=1}^{c} \sum_{k=1}^{N} u_{i k}^{m}\left\|X_{k}-V_{i}\right\|^{2}
$$

Where $x_{k}$ is the gray value of the $k$ th, $v_{i}$ is the fuzzy cluster center of the $i$ th cluster, $u_{i k}$ represents the membership of $k$ th pixel in the $i$ th cluster, $\mathrm{c}$ is the number of clusters, $\mathrm{N}$ is the number of data points, and $\mathrm{m}$ is weighting exponent which determines the fuzziness of the resulting segmentation.

The fuzzy membership function and clustering center function are updated as follows:

$$
\begin{aligned}
& u_{i k}=\frac{\left(\left\|x_{k}-v_{i}\right\|^{2}\right)^{-\frac{1}{(m-1)}}}{\sum_{j=1}^{c}\left(\left\|x_{k}-v_{j}\right\|^{2}\right)^{-\frac{1}{(m-1)}}} \\
& v_{i}=\frac{\sum_{k=1}^{n} u_{i k}^{m} x_{k}}{\sum_{k=1}^{n} u_{i k}^{m}}
\end{aligned}
$$

The standard FCM proceeds by iterating two necessary conditions until $\left|V_{\text {new }}-V_{\text {old }}\right|<\varepsilon$, where $\varepsilon$ is a termination condition between 0 and 1 . Each data point will be associated with a membership value for each cluster after the FCM clustering. By assigning the data point to the cluster with the highest membership value, a segmentation of the image could be obtained.

In the standard FCM, pixels of an image are assumed to be independent of each other and the spatial information is not taken into consideration. However, neighboring pixels in an object usually have strong correlation. Thus, the incorporation of spatial information between adjacent pixels based on the standard FCM can produce more meaningful classification.

\section{FCM_S Algorithm}

To improve the clustering results of FCM, spatial information is incorporated into the standard FCM algorithm. The theory of incorporating spatial information is that neighboring pixels have similar feature values, and the probability is great that they 
belong to the same cluster. To utilize the spatial information, the membership function is usually modified as follows [12]:

$h_{i k}=\frac{1}{N_{R}} \sum_{k \in N_{k}} u_{i k}$

Where $N_{k}$ represents the set of neighboring pixels within a window around $x_{k} \cdot N_{R}$ is the size of set $N_{k}$. The spatial function $h_{i k}$ represents the possibility that pixel $x_{i}$ belongs to kth cluster.

The membership function incorporating spatial function is modified as follows:

$$
u_{i k}=\frac{u_{i k}^{p} h_{i k}^{q}}{\sum_{j=1}^{c} u_{j k}^{p} h_{j k}^{q}}
$$

$\mathrm{p}$ and $\mathrm{q}$ are parameters to determine the importance of both functions. The adjacent pixels have similar feature values. If the majority of neighboring pixels belong to the same cluster, the value of spatial function for a pixel will be enhanced. On the contrary, if a pixel is an outlier, the value of spatial function for the pixel will be reduced by the values of its neighboring pixels.

The FCM_S clustering is a two-step process. In the first process, the membership function is calculated in the gray levels just like the standard FCM. In the second process, the membership information of each pixel is mapped to the spatial domain by Equation (5).

However, the spatial information is not considered in the first process of the FCM_S algorithm. In addition, the FCM_S algorithm only works on grayscale images. Therefore, the FCM_S algorithm is not suitable for color image segmentation.

\section{The nFCM_S Algorithm Incorporating Spatial Information for Color Image Segmentation}

\subsection{HSV Color Space}

In the FCM_S algorithm, gray information is only considered as the input so that some details of color information may be lost. When the contrast of grayscale image is low and the target is complex, the over-segmentation will be severe. To improve segmentation performance, HSV color space is used for decomposition of color image. Therefore, the color image needs to be transformed from RGB color space to HSV space. In a RGB color space, $\mathrm{r}, \mathrm{g}$, and $\mathrm{b}$ are the red, green, and blue coordinates respectively that we extract directly from color images. And then we let max be the greatest of r, g, and b, and min be the least of $r, g$, and $b$. To get the hue angle $h[0,360]$ for HSV space, we compute as follows: 


$$
h=\left\{\begin{array}{l}
0 \quad \text { if } \max =\min \\
\left(60^{\circ} \times \frac{g-b}{\max -\min }+0^{\circ}\right) \bmod 360^{\circ}, \quad \text { if } \max =b \\
60^{\circ} \times \frac{b-r}{\max -\min }+120^{\circ},
\end{array}\right.
$$

To get saturation s for HSV space, we compute as follows:

$$
s= \begin{cases}0, & \text { if } \max =0 \\ \frac{\max -\min }{\max }=1-\frac{\min }{\max }, & \text { otherwise }\end{cases}
$$

And to get saturation v for HSV space, we compute as follows:

$v=\max$

\subsection{A Linearly-Weighted Sum Image for HSV Color Space}

In order to label pixels accurately, we modify the input of FCM_S. Before the clustering iterative process, a linearly-weighted sum image $\eta_{k}[14]$ is computed to relabel a pixel with its adjacent pixel information.

The input value of each HSV component is modified as follows:

$$
\eta_{k}=\frac{1}{1+\beta}\left(x_{k}+\frac{\beta}{N_{R}} \sum_{j \in N_{k}} x_{j}\right)
$$

Where $x_{k}$ is each HSV component value of the kth pixel. $N_{k}, N_{R}$ are defined as before. $\beta$ is the linearly-weighted parameter. Apparently, all of these improve adaptively to the local image information. A linearly-weighted sum image of each HSV component can take into fully consideration of the adjacent pixels information and enable the labeling of a pixel to be associated with its neighborhood. Therefore, the pixels can be relabeled to group them effectively into suitable clusters.

\subsection{A Novel FCM with Spatial Information}

To improve the clustering segmentation results, in the first process of FCM_S, the spatial-based image segmentation is incorporated, the objective function in Equation (1) is modified as follows:

$$
J_{n F C M_{-} S}=(1-\alpha) \sum_{i=1}^{c} \sum_{k=1}^{N} u_{i k}^{m}\left\|\eta_{k}-v_{i}\right\|^{2}+\frac{\alpha}{N_{R}} \sum_{i=1}^{c} \sum_{k=1}^{N} u_{i k}^{m} \sum_{r \in N_{k}}\left\|x_{r}-v_{i}\right\|^{2}
$$

As we can see from Equation (10), there are two terms in the objective function. The first term weighted by $(1-\alpha)$ in Equation (10) stands for the pixels information. The 
second term weighted by $\alpha$ imposes the set of neighboring pixels information. Apparently, the crucial parameter $\alpha$ is assigned to control the weight of neighboring pixels and it can keep a better balance between the center pixel and its neighboring pixels. Our optimal weighting scheme formulates a spatial constraint to keep spatial continuity around $x_{k}$.

According to the optimal objective functions, the membership function $u_{i k}$ and cluster center function $v_{i}$ are modified in Equations (11)-(12):

$$
\begin{aligned}
& u_{i k}=\frac{\left((1-\alpha)\left\|\eta_{k}-v_{i}\right\|^{2}+\frac{\alpha}{N_{R}} \sum_{r \in N_{k}}\left\|X_{r}-v_{i}\right\|^{2}\right)^{-\frac{1}{(m-1)}}}{\sum_{j=1}^{c}\left((1-\alpha)\left\|\eta_{k}-v_{j}\right\|^{2}+\frac{\alpha}{N_{R}} \sum_{r \in N_{k}}\left\|X_{r}-v_{i}\right\|^{2}\right)^{-\frac{1}{(m-1)}}} \\
& v_{i}=\frac{\sum_{k=1}^{n} u_{i k}^{m}\left((1-\alpha) \eta_{k}+\frac{\alpha}{N_{R}} \sum_{r \in N_{k}} x_{r}\right)}{\sum_{k=1}^{n} u_{i k}^{m}}
\end{aligned}
$$

As can be seen from Equations (11) and (12), there are also two terms in the improved $u_{i k}$ and $v_{i}$ to utilize spatial information comprehensively and achieve clustering better, just like the modified Equation (10).

The nFCM_S algorithm is also a two-step process. In the first process, we separately compute the linearly-weighted sum on each component of the HSV model, and then we applied the nFCM_S algorithm on each component of the HSV model to calculate initial fuzzy membership and cluster center incorporating spatial information. In the second process, the membership information of each pixel is mapped to the spatial domain, and the spatial function is computed from that. The nFCM_S iteration proceeds with the new fuzzy membership and cluster center that is incorporated with the spatial function. The iteration is stopped when the maximum difference between two cluster centers at two successive iterations is less than a threshold (0.01). After the convergence, each pixel is assigned to a specific cluster for which the membership is maximal.

Figure 1 shows the flowchart of the nFCM_S algorithm. The proposed nFCM_S algorithm has several main steps.

Step1. Input the RGB color space of original images, and transform the RGB color space to HSV model. The linearly-weighted sum of each HSV component is computed with Equation (9).

Step2. Set the number c of clusters for different color images. The initial clustering centers are initialized randomly. 


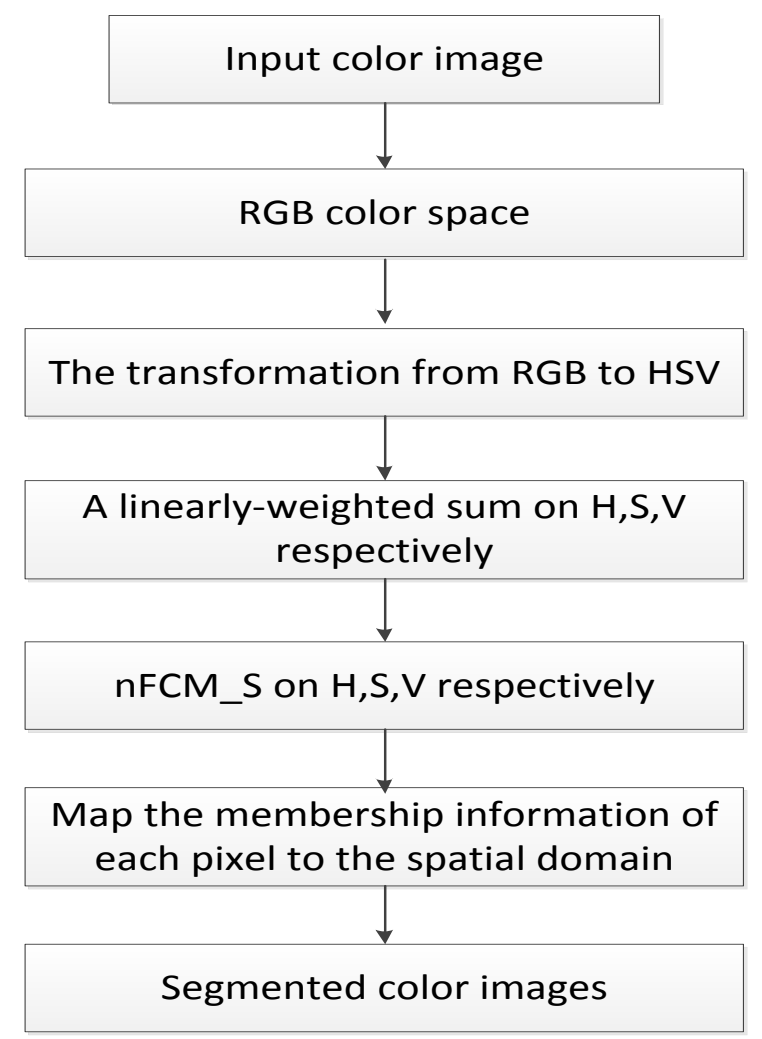

Figure 1. Flowchart of the nFCM_S Algorithm

Step3. Segment the color image according to (11) and (12) to obtain the initial fuzzy membership function $u_{i k}$ and clustering center $v_{i}$.

Step4. Update the fuzzy membership function $u_{i k}$ and clustering center $v_{i}$ with Equations (5) and (12).

Repeat Step 4 until the following termination criterion is satisfied:

$\left|V_{\text {new }}-V_{\text {old }}\right|<\varepsilon$

\section{Experimental Results}

In order to test the color image segmentation performance, the experimental results of our proposed nFCM_S algorithm is compared with the standard FCM and the FCM_S proposed in [12]. A window of $3 * 3$ is used in this paper, and the other parameters are: $\mathrm{m}=2, \quad N_{R}=8, \varepsilon=0.01, \mathrm{p}=1, \mathrm{q}=1$.

\subsection{Comparison of Segmentation Results on Color Images}

Figure 2, illustrates four color nature scene images we tested. Here, for Figure 2(a), Figure 2(b), Figure 2(c), Figure 2(d), the parameter $c=2, c=2, c=2, c=3$ are respectively used in the experiment. We performed comparisons of the segmentation results with different segmentation algorithms in Figures 2-5, respectively. 


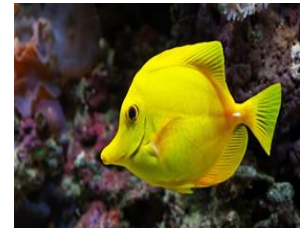

(a)

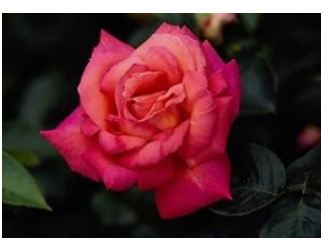

(b)

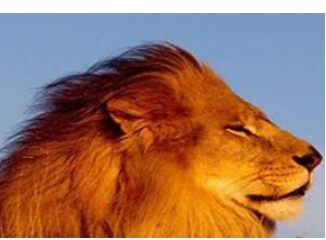

(c)

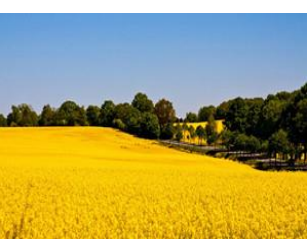

(d)

Figure 2. The Color Images

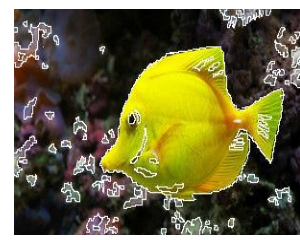

(a)

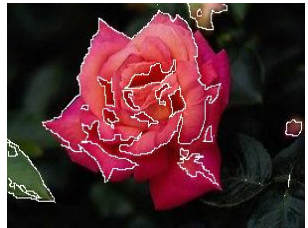

(b)

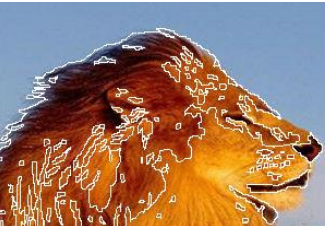

(c)

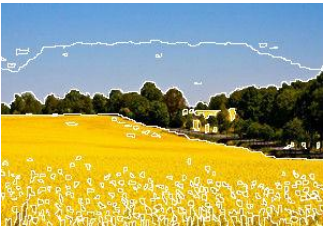

(d)

Figure 3. Segmentation Results with the Standard FCM Algorithm

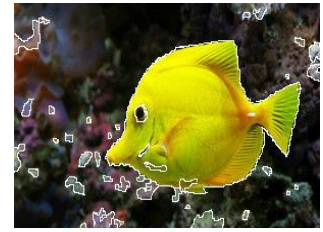

(a)

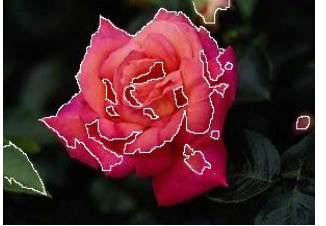

(b)

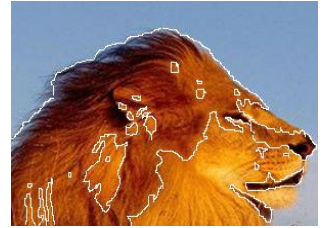

(c)

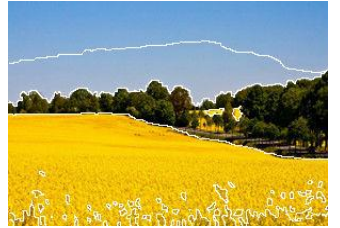

(d)

Figure 4. Segmentation Results with the FCM_S Algorithm in [12]

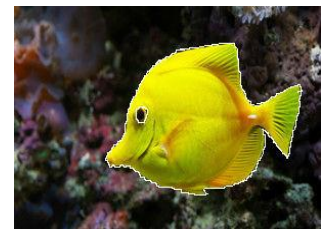

(a)

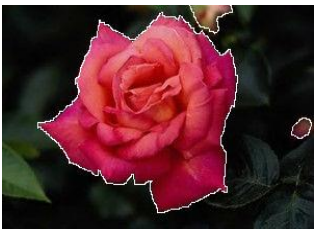

(b)

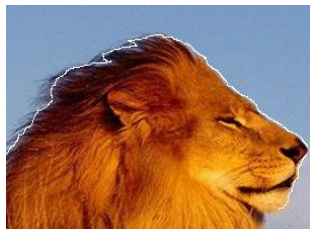

(c)

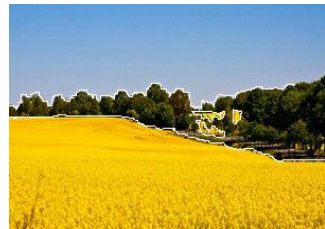

(d)

Figure 5. Segmentation Results with the Proposed nFCM_S Algorithm

The segmentation results with the standard FCM algorithm and the FCM_S algorithm are shown in Figures 3-4. Figure 3, and Figure 4, are obviously over-segmented, and some regions are inappropriately merged into the same cluster. The segmentations in Figure 5, demonstrate that the nFCM_S algorithm not only succeeds in segmenting color images but also shows better segmentation and produces more detailed and accurate boundaries.

\subsection{Comparison of Quality Evaluation with Different Algorithms}

Partition coefficient (PC) and classification entropy (CE) are two cluster validity functions to evaluate segmentation accuracy and validity in different clustering methods. The higher value of PC means the greater performance. Oppositely, the lower value of CE means the better performance.

As a result, the best clustering is achieved when the value of PC is maximal or CE is minimal. Functions of PC and CE are as follows:

$$
P C=\frac{\sum_{j}^{N} \sum_{i}^{c} u_{i j}^{2}}{N}
$$




$$
C E=\frac{-\sum_{j}^{N} \sum_{i}^{c}\left(u_{i j} \log u_{i j}\right)}{N}
$$

Table 1. The Comparison of PC and CE with Different Algorithms for Several Color Images

\begin{tabular}{|l|l|l|l|l|}
\hline & & FCM & FCM_S in[12] & nFCM_S \\
\hline \multirow{2}{*}{ Image 1 } & PC & 0.7413 & 0.8625 & 0.9250 \\
\cline { 2 - 5 } & CE & 0.0103 & 0.0083 & 0.0059 \\
\hline \multirow{2}{*}{ Image 2 } & PC & 0.7014 & 0.8516 & 0.9235 \\
\cline { 2 - 5 } & CE & 0.1255 & 0.0080 & 0.0074 \\
\hline Image 3 & PC & 0.6496 & 0.8429 & 0.9342 \\
\cline { 2 - 5 } & CE & 0.1460 & 0.0067 & 0.0045 \\
\hline Image 4 & PC & 0.7973 & 0.8086 & 0.8757 \\
\cline { 2 - 5 } & CE & 0.0379 & 0.0094 & 0.0014 \\
\hline
\end{tabular}

From the Table 1, we observe that the nFCM_S algorithm can get higher PC value and lower CE value, and the performance of the nFCM_S algorithm is better than the other methods for color image segmentation.

\section{Conclusions}

In this paper, we have proposed a novel fuzzy c-means algorithm (nFCM_S) incorporating local spatial information and color information for color image segmentation. A linearly-weighted sum on each HSV component based on the standard FCM algorithm has been proposed. In addition, the optimized fuzzy clustering algorithm using spatial information has got better segmentation results for color images. Experimental results show that the nFCM_S algorithm is appropriate for color image segmentation. In the future, we plan to extend our algorithm to image retrieval for larger datasets and other applications.

\section{Acknowledgments}

This work is partially supported by National Nature Foundation (61371092) and National Nature Science Foundation (61071074) and Jilin Province Nature Foundation (201215016).

\section{References}

[1] Y. Zhuge and J. K. Udupa, "Intensity standardization simplifies brain MR image segmentation", Computer vision and image understanding, vol.113, no.10, (2009), pp. 1095-1103.

[2] M. Daliri, H. A. Moghaddam, S. Ghadimi, M. Momeni, F. Harirchi and M. Giti, "Skull segmentation in 3D neonatal MRI using hybrid Hopfield Neural Network", in: Proceedings of the IEEE International Conference on EMBC, (2010), pp. 4060-4063.

[3] D. J. Hemanth, C. K. S. Vijila, A. I. Selvakumar and J. Anitha, "Distance metric-based time-efficient fuzzy algorithm for abnormal magnetic resonance brain image segmentation", Neural Computing and Applications, vol. 22, no. 5, pp. 1013-1022.

[4] B. Tanoori, Z. Azimifar, A. Shakibafar and S. Katebi, "Brain volumetry: an active contour model-based segmentation followed by SVM-based classification", Comput.Biol.Med, vol. 41, no. 8, (2011), pp. 619-632.

[5] M. Antonios, G. S. Ioannis, L. Christian, A. Paul, S. Ahmed, H. V. Joseph, E. A. David, C. J. Serena and R. Daniel, "Automatic Whole Brain MRI Segmentation of the Developing Neonatal Brain", IEEE Transactions on medical imaging, vol. 33, (2014) September, pp.1818-1831. 
[6] Y. Wen, L. He and K. M. V. Deneen, "Brain tissue classification based on DTI using an improved Fuzzy C-means algorithm with spatial constraints", Magnetic Resonance Imaging, vol. 31, no. 9, (2013) November, pp.1623-1630.

[7] T. Saikumar, M. Nagarani, K. Yojana and B. Shashidhar, "Image Segmentation of an Adaptive Threshold Algorithm Using Watershed Transform and Fuzzy C-Means Clustering on Level Set Method", IEEE-International Conference On Advances In Engineering, Science And Management, (2012) March, pp. 33-38.

[8] M. A. Balafar, "Fuzzy C-mean based brain MRI segmentation algorithms", Artificial Intelligence Review, vol. 41, no. 3, (2014) March, pp.441-449.

[9] E. Nadernejad and S. Sharifzadeh, "A new method for image segmentation based on Fuzzy C-means algorithm on pixonal images formed by bilateral filtering", Signal, Image and Video Processing, vol. 7, no. 5, (2013) September, pp 855-863.

[10] P. Kumar and D. Sirohi, "Comparative Analysis of FCM and HCM Algorithm on Iris Data Set", International Journal of Computer Applications, vol. 5, no. 2, (2010) August.

[11] W. Cai, S. Chen and D. Q. Zhang, "Fast and robust fuzzy c-means clustering algorithms incorporating local information for image segmentation", Pattern Recognition, vol. 40 (2007), pp. 825-838.

[12] L. He, Y. Wen, M. Wan and S. Liu, "Multi-channel features based automated segmentation of diffusion tensor imaging using an improved FCM with spatial constraints", Neurocomputing, vol. 137, (2014), pp.107-114.

[13] J. C. Bezdek, "Pattern Recognition with Fuzzy Objective Function Algorithms", Plenum, New York, (1981).

[14] L. Szilágyi, Z. Benyó, S. M. Szilágyii and H. S. Adam, "MR brain image segmentation using an enhanced fuzzy c-means algorithm", in: $25^{\text {th }}$ Annual International Conference of IEEE EMBS, (2003), pp. 17-21.

\section{Authors}

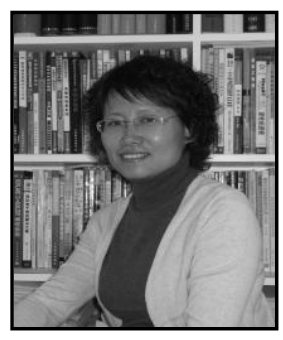

Li Ling, (1965), female, master, associate professor, research areas: intelligent information processing, networking protocol, cloud computing

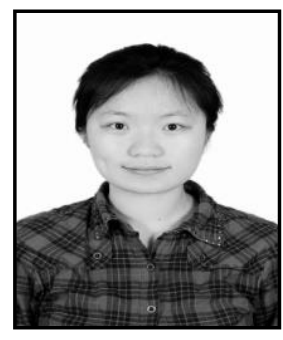

Song Yingwei, (1991), female, master, postgraduate, research areas: image processing, intelligent information processing 
International Journal of Signal Processing, Image Processing and Pattern Recognition Vol. 9, No. 8 (2016) 